\title{
Melanoma Research
}

\section{The successful use of Pembrolizumab in a renal transplant recipient with metastatic melanoma \\ --Manuscript Draft--}

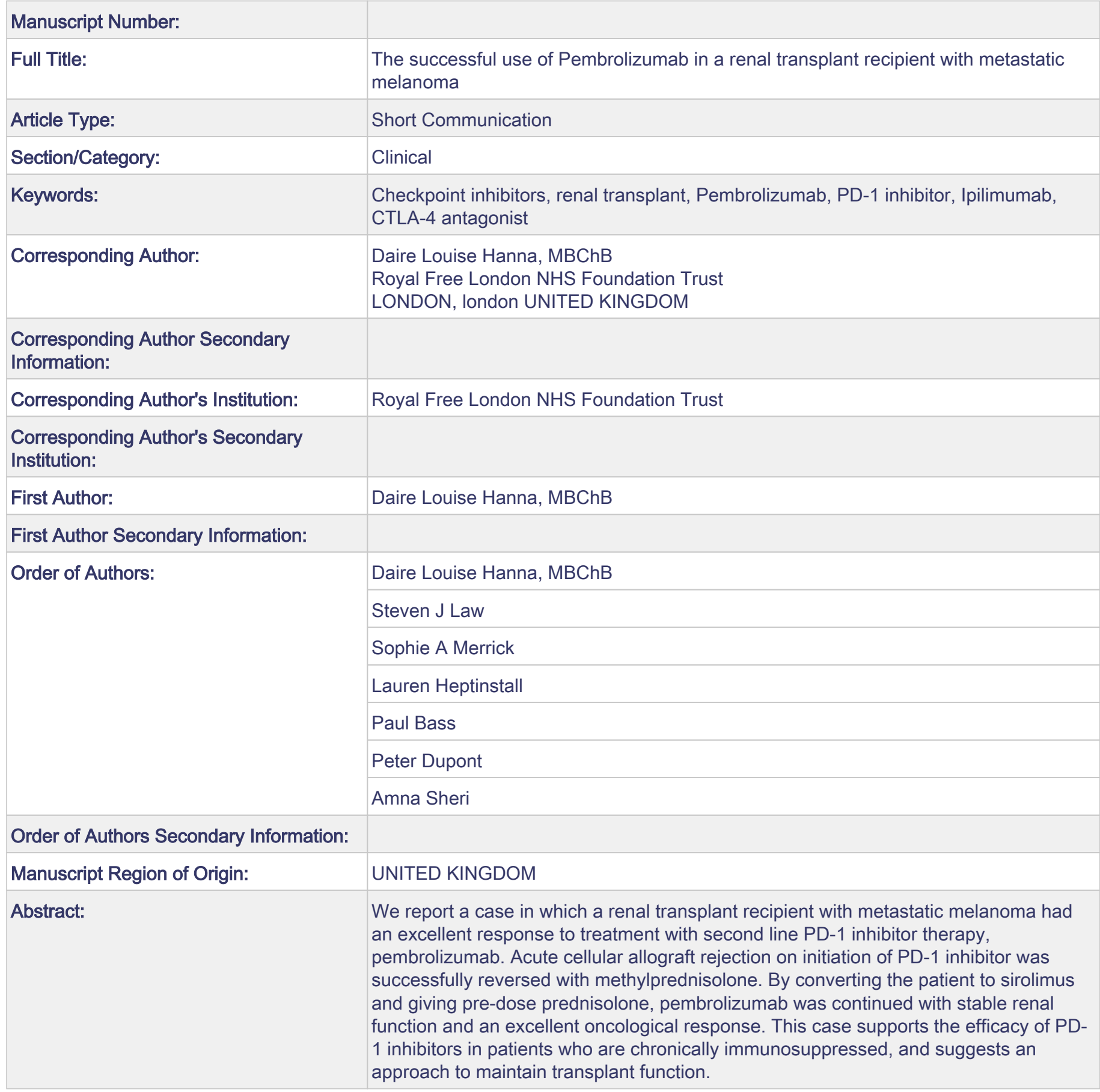


The successful use of Pembrolizumab in a renal transplant recipient with metastatic melanoma

Short title: Pembrolizumab use in a transplant patient

DL Hanna*, SJ Law*, SA Merrick, L Heptinstall, P Bass, P Dupont, A Sheri *joint first authors

Royal Free Hospital, Pond Street, Hampstead, London, NW3 2QG

Corresponding author: Daire L Hanna. Royal Free Hospital, Pond Street, Hampstead, London, NW3 2QG

Conflicts of interest: none declared 


\section{Abstract}

We report a case in which a renal transplant recipient with metastatic melanoma had an excellent response to treatment with second line PD-1 inhibitor therapy, pembrolizumab. Acute cellular allograft rejection on initiation of PD-1 inhibitor was successfully reversed with methylprednisolone. By converting the patient to sirolimus and giving pre-dose prednisolone, pembrolizumab was continued with stable renal function and an excellent oncological response. This case supports the efficacy of PD-1 inhibitors in patients who are chronically immunosuppressed, and suggests an approach to maintain transplant function.

Key words: Checkpoint inhibitors, renal transplant, Pembrolizumab, PD-1 inhibitor, Ipilimumab, CTLA-4 antagonist 


\section{Introduction}

Anti-cytotoxic T lymphocyte-associated antigen protein 4 (CTLA-4) and programmed cell death protein 1 (PD1) inhibitors have transformed long term outcomes in metastatic melanoma. At present there are no guidelines in place for using checkpoint inhibitors (CPIs) in patients with solid organ transplants as this cohort was excluded from trials involving checkpoint inhibitory antibodies. Our knowledge on the risk of acute allograft rejection in these patients is limited to a small number of case reports.

We report a case in which a renal transplant patient with metastatic melanoma had an excellent response to treatment with second line PD-1 inhibitor therapy, pembrolizumab. Acute cellular allograft rejection on initiation of PD-1 inhibitor was successfully reversed with methylprednisolone. The patient's immunosuppression regime was changed to a combination of tacrolimus, sirolimus and prednisolone triple therapy, which allowed pembrolizumab to be continued with stable renal function. This case supports the efficacy of PD-1 inhibitors in patients who are chronically immunosuppressed.

\section{Case Report}

A 52-year-old man with end stage renal failure secondary to $\lg$ A nephropathy and hypertension underwent a pre-emptive kidney transplant from an altruistic donor in June 2013. In July 2018 he was diagnosed with metastatic melanoma with pulmonary nodules. Biopsy confirmed wild type BRAF mutation status on primary tumour. He was ECOG performance status zero at the time of diagnosis and his immunosuppression regime entailed double agent tacrolimus ( $2 \mathrm{mg}$ twice daily) and mycophenolate mofetil (500mg twice daily).

On diagnosis he was commenced on CTLA-4 antagonist, ipilumumab. At this point tacrolimus dose was reduced by $25 \%$ (1.5mg twice daily) and mycophenolate mofetil (MMF) dose was reduced by $50 \%$ ( $250 \mathrm{mg}$ twice daily). 
After two months of treatment with ipilumumab the patient developed a worsening cough, which prompted a CT chest to be performed to investigate for pneumonitis. The CT, although an early scan, demonstrated unequivocal disease progression with clear radiological progression of disease with new lung lesions. Although restaging scans are usually performed after three months of treatment it was felt that the clinical picture combined with the radiological evidence of disease progression necessitated stopping first line treatment. Blood tests were taken weekly and the patient's renal function remained stable throughout treatment with ipilumumab.

Second line treatment with PD-1 inhibitor, pembrolizumab was commenced in December 2018 following extensive counselling on the possible adverse effects including acute allograft rejection.

Creatinine prior to commencing pembolizumab was $107 \mathrm{mcmol} / \mathrm{L}$. Acute deterioration in his renal function was noted four days post commencing treatment with creatinine rising to $150 \mathrm{mcmol} / \mathrm{L}$. He was asymptomatic with normal observations and a negative urine dip for leucocytes, nitrites, protein and haematuria; biochemistry showed normal electrolytes, liver function, bone profile and inflammatory markers, trough tacrolimus level was 5; cytomegalovirus and BK polyomavirus PCR in blood was negative. Renal transplant ultrasound demonstrated an unobstructed kidney with resistive indices of 0.63-0.71.

Creatinine rose further to $184 \mathrm{mcmol} / \mathrm{L}$ and a renal allograft biopsy was performed (Fig.1). The biopsy showed a diffuse inflammatory infiltrate containing predominantly lymphocytes, with plasma cells and a few eosinophils in areas. Acute tubular damage with ragged cytoplasm and nuclear pleomorphism was noted and there was extensive infiltration of the tubules by lymphocytes. There were no glomerular or vascular changes. No vascular rejection was present. A histological diagnosis of Banff $1 \mathrm{~b}$ acute cellular rejection was made although it was noted the appearances were hard to distinguish from acute interstitial nephritis. 
He was treated with three doses of pulsed IV Methylprednisolone $500 \mathrm{mg}$ followed by prednisolone $20 \mathrm{mg}$ daily. At this point the MMF dose was increased to $500 \mathrm{mg}$ twice daily and tacrolimus was continued at dose $1.5 \mathrm{mg}$ twice daily.

In one months' time his creatinine had stabilised at $110 \mathrm{mcmol} / \mathrm{L}$ from a peak of $206 \mathrm{mcmol} / \mathrm{L}$. Prior to cycle two, MMF was stopped; tacrolimus dose was increased to $2 \mathrm{mg}$ twice daily and sirolimus $5 \mathrm{mg}$ once daily was started.

The steroid regime we use involves increasing the prednisolone dose to $40 \mathrm{mg}$ on the day of pembrolizumab infusion, maintaining this dose for one week and then tapering it to a $5 \mathrm{mg}$ maintenance dose. Initially we used higher maintenance doses (20mg prednisolone after cycles one and two) but have been able to titrate this dose down without detriment to the patient's renal function.

The patient has now tolerated eight cycles of pembrolizumab with stable creatinine. A restaging CT scan showed an excellent treatment response with marked reduction in size of the pulmonary metastases (largest lesion $18 \mathrm{~mm}$ reduced to $3 \mathrm{~mm}$, see fig 2 ) and the single bone lesion no longer visualised (replaced with area of sclerotic focus). His ECOG performance status has remained zero. A recent scan after five months of treatment shows an ongoing treatment response.

\section{Discussion}

Checkpoint inhibitors destroy malignant cells by increasing T cell activation. By the same mechanism kidney allograft donor antigens stimulate $T$ cell activation, which can result in acute cellular rejection. Immune related adverse events are significantly less common in the use of PD- 1 inhibitors antibodies compared with CTLA-4 antagonists such as ipilumumab. Of the limited available case reports on transplant patients, there is support for the use of CTLA4 antagonists over PD-1 inhibitors as they are associated with a lesser risk of transplant rejection. This is what drove our decision to commence ipilumumab as first line treatment.(1) 
Concerns of increased T cell activation have limited the use of these therapies in transplantation due to the potential reduction in immune tolerance to the allograft. However, given the lack of efficacious alternative treatments increasing numbers of cases are being reported.

A recent retrospective analysis by Abdel-Whab et al looked at the safety of checkpoint inhibitors to treat cancer in solid organ transplant recipients.

Their data showed a high allograft rejection rate shortly after initiating checkpoint inhibitors, which was accompanied by high mortality rates. Of the 39 patients with a variety of tumour types, 23 had renal transplants. Four patients were treated with second line CPI (ipilumamab followed by nivolumab or pembolizumab). Of these, $50 \%$ suffered acute renal allograft rejection. 7 out of 22 melanoma patients with a solid organ transplant (renal, cardiac or liver) had a partial or complete response to treatment. Tumour response was found to be less frequent in the patients with allograft rejection.(2)

Aguirre et al reported 20 cases of checkpoint inhibitor use with solid organ allografts which showed rejection in 8 out of 11 of patients treated with nivolumab, 2 out of 2 patients treated with pembrolizumab and 0 out of 4 in those treated with ipilimumab.(3) Focussing on kidney transplantation, Perazella et al showed acute rejection in 1 out of 3 patients treated with ipilimumab and 8 out of 14 treated with PD-1 inhibitors. The risk of rejection is further increased by the common practice of reducing immunosuppression upon diagnosis of malignancy.(4)

Lipson et al reported a case in which there was renal allograft failure post treatment with PD-1 antagonist. The excised kidney was described as containing many infiltrating PD-1-positive T cells and PD-L1-positive and PD-L2-positive endothelial cells which is consistent with cell mediated rejection induced by blockade of the PD-1 pathway.(5) Our patient experienced acute cellular allograft rejection on initiation of treatment with PD-1 inhibitor. He responded well to treatment with methylprednisolone with renal function recovering to baseline. Due to the lack 
of literature surrounding recommencing a checkpoint inhibitor post recovery from allograft rejection we were guided by recent case reports.

The successful use of PD1-inhibitors in renal transplant patients has been reported in a few cases including patients with cutaneous squamous cell carcinoma, duodenal cancer and urothelial cancer. These cases support the use of sirolimus and high dose prednisolone $(5 \mathrm{mg} / \mathrm{kg})$ for one-week pre pembrolizumab dose.(6,7) We believe that by commencing sirolimus (mTOR inhibitor) in combination with pre-treatment steroids, our patient was able to maintain a stable renal function while continuing treatment with PD-1 inhibitor.

Real world practice suggests that the efficacy of PD-1 inhibitors is unlikely to be affected by glucocorticoid use when used to treat immune related adverse events. We have less of an understanding of the impact of the efficacy of checkpoint inhibitors in chronic immunosuppression. Recent research suggests that baseline corticosteroid use (prednisolone $>10 \mathrm{mg}$ daily) in previously PD-1 inhibitor naïve patients can have a negative impact on disease-free and overall survival.(8) This case supports the use of PD-1 inhibitors in chronic immunosuppression.

The efficacy of the combination of a glucocorticoid and sirolimus in preventing immunerelated adverse events associated with PD-1 inhibitors is not known. In certain tumour types, such as renal cell carcinoma and Kaposi's sarcoma, it has been hypothesized that sirolimus has the potential to act as an immunosuppressing agent while simultaneously exhibiting antitumour properties.(7) In animals their combination resulted in a more durable and synergistic tumour regression compared to either agent being used alone. $(7,9)$ In the treatment of melanoma, sirolimus is unlikely to enhance pembrolizumab's action on malignant cells. However it seems preferable to use sirolimus in place of the antimetabolite (MMF) for this reason.

In native kidneys checkpoint inhibitors cause an acute interstitial nephritis. Histologically this is indistinguishable from acute cellular rejection and the treatment is glucocorticoids. The role of steroids in this case may simply be to reduce an allergic interstitial nephritis in the 
transplanted kidney, rather than impede allorecognition. An alternative for the high incidence of acute rejection with checkpoint inhibitors is that CTLA-4 inhibitors provide a co-stimulatory signal in the context of graft allorecognition by the CD4 T cell and the PD1 inhibitor enhances the cytotoxicity of the effector (CD8) arm of the allo-immune response. In this setting we would not expect steroids alone to be effective. This is the rational for decreasing $T$ cell activation with tacrolimus and sirolimus.

\section{Conclusion}

It is possible to successfully treat metastatic melanoma with PD-1 inhibitor therapy in renal transplant recipients. Acute rejection episodes are common but can be managed without graft loss while continuing PD-1 inhibitor therapy.

This case supports the use of sirolimus and prednisolone to preserve transplant function during PD-1 inhibitor therapy. The role of sirolimus in this is uncertain. We suspect the key element is the use of steroids in preventing interstitial inflammation in the graft rather than to damp down a specific anti-graft allo-immune response. The absence of $\mathrm{C} 4 \mathrm{~d}$ on the transplant biopsy supports this.

Furthermore this case provides evidence for the efficacy of PD-1 inhibitors in chronically immunosuppressed patients. It also highlights the importance of a multidisciplinary team approach in discussing benefits and risks of treatment with the patient. 
Figure 1. A) Haematoxylin and Eosin low power (x200) demonstrating a diffuse predominantly lymphocytic inflammatory infiltrate and acute tubular damage B) Periodic AcidMethenamine Schiff stain at low power (x200) showing lymphocytic infiltration of tubular epithelial cells, C) CD3 immunohistochemistry highlighting lymphocytes (x200) All in-keeping with a histological diagnosis of Banff $1 b$ acute cellular rejection.

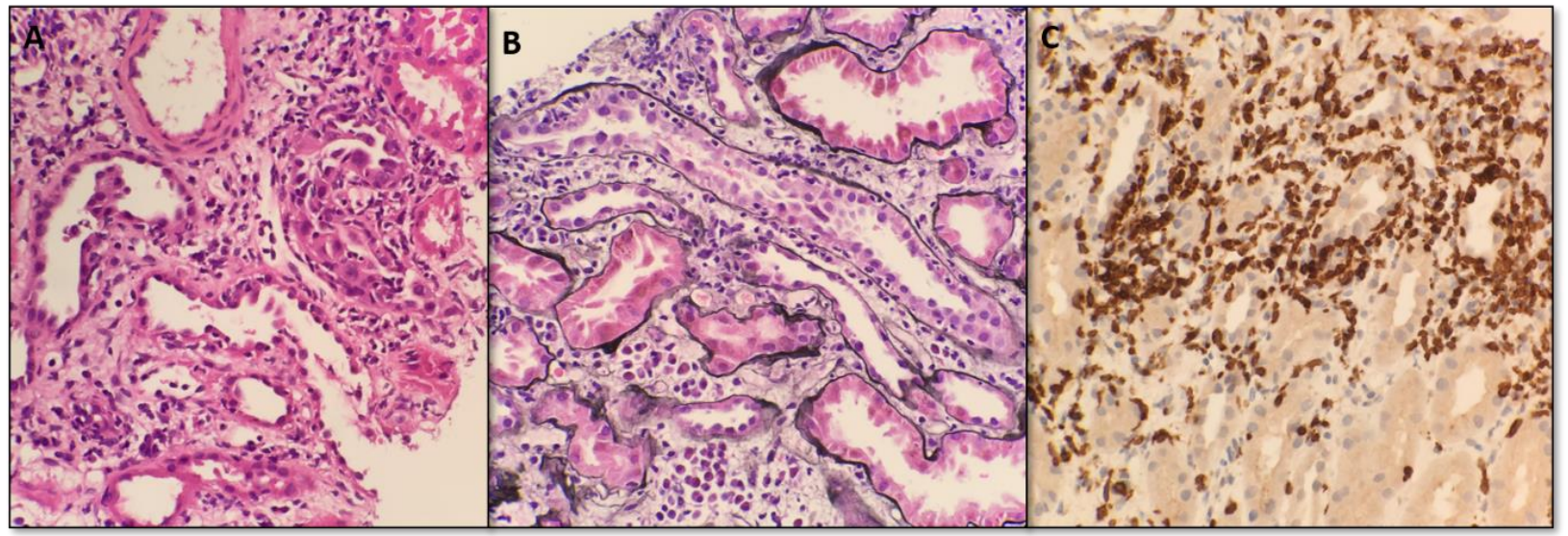

Figure 2. A) CT chest pre pembrolizumab, B) CT chest five months after initiation of pembrolizumab.

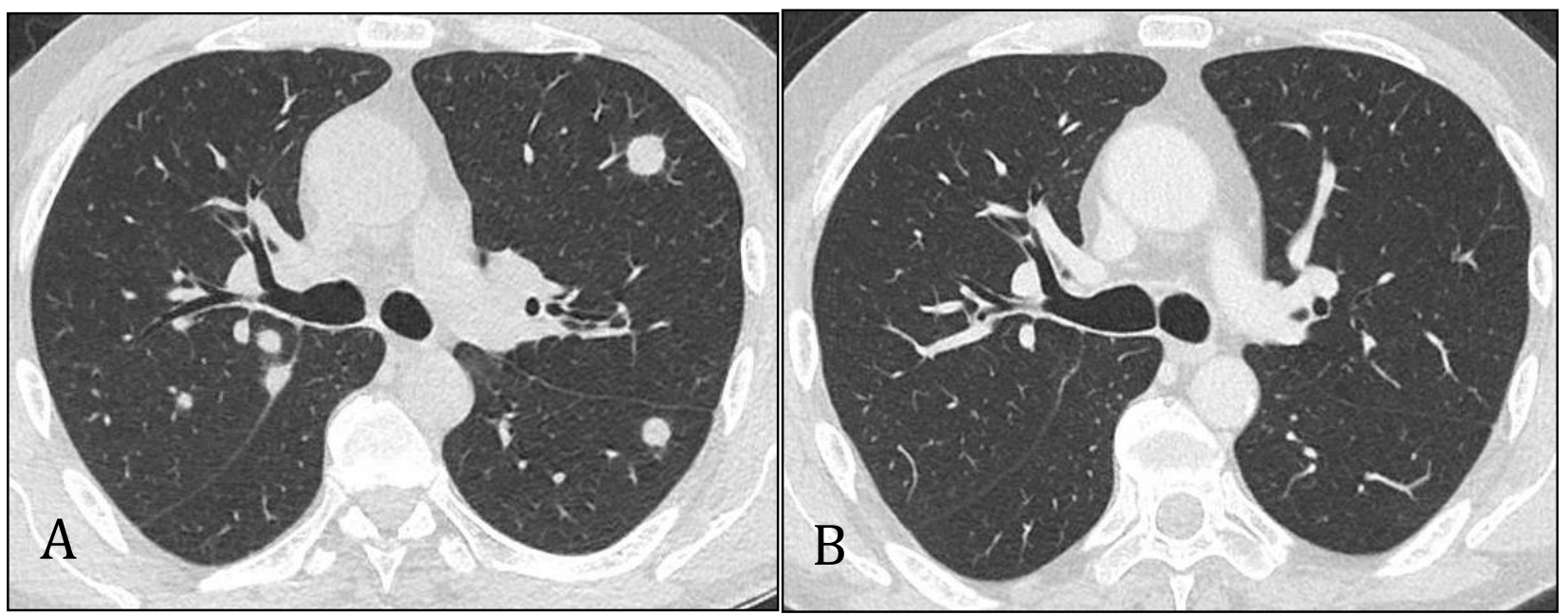




\section{References}

(1) Kwatra V, Karanth NV, Priyadarshana K, Charakidis M. Pembrolizumab for metastatic melanoma in a renal allograft recipient with subsequent graft rejection and treatment response failure: a case report, J med Case Rep 2017, 11 (73): 1-5.

(2) Abdel-Wahab N, Safa H, Abudayyeh A, Johnson DH, Trinh VA, Zobniw CM, Lin H et al. Checkpoint inhibitor therapy for cancer in solid organ transplantation recipients: an institutional experience and a systematic review of the literature, $\mathrm{J}$ Immunother Cancer 2019, 7(106): 1-10.

(3) Aguirre LE, Guzman ME, Lopes G, Hurley J. immune Checkpoint Inhibitors and the Risk of Allograft Rejection: A Comprehensive Analysis on an Emerging Issue, Oncologist 2019, 24(3): 394-401

(4) Perazella MA, Shirali AC. Nephrotoxicity of Cancer Immunotherapies: Past, Present and Future, J Am Soc Nephrol 2018, 29 (8): 1-14.

(5) Lipson EJ, Bagnasco SM, Moore J, Jang S, Patel MJ, Zachary AA et al. Tumor Regression and Allograft Rejection after Administration of Anti-PD-1, N Eng J Med 2016, 374 (9): $896-898$

(6) Wu CK, Juang GD, Lai HC. Tumor regression and preservation of graft function after combination with anti-PD-1 immunotherapy without immunosuppressant titration, Ann Oncol 2017, 28 (11): 2895-2896.

(7) Barnett R, Barta VS, Jhaveri KD. Preserved Renal-Allograft Function and the PD-1 Pathway Inhibitor Nivolumab, N Engl J Med 2017, 376 (2): 191-192 
(8) Arbour KC, Mezquita L, Long N, Rizvi H, Auclin E, Ni A et al. Impact of Baseline Steroids on Efficacy of Programmed Cell Death-1 and Programmed Death-Ligand 1 Blockade in Patients With Non-Small-Cell Lung Cancer, J Clin Oncol 2018, 36 (28): 2872-2878

(9) M Sadaat, S Jang. Complete Tumor Response to Pembrolizumab and Allograft Preservation in Renal Allograft Recipient on Immunosuppressive Therapy, J Oncol Pract 2018, 14 (3): 198-199 